\title{
Data Analytic Tool for Clustering Identification based on Dimensionality Reduction of Frequency Measurements
}

\author{
Felix Rafael Segundo Sevilla \\ Petr Korba \\ ZHAW \\ Winterthur, Switzerland
}

\author{
Emilio Barocio \\ $\mathrm{U}$ de $\mathrm{G}$ \\ Guadalajara, México
}

\author{
Hector Chavez \\ USACH \\ Santiago de Chile, Chile
}

\author{
Walter Sattinger \\ Swissgrid \\ Aarau, Switzerland
}

\begin{abstract}
This work presents a data analytic tool for clustering analysis based on Dimensionality Reduction (DR) of power system measurements. The proposed method is applied to frequency measurements of the ENTSO-E dynamic model of continental Europe and the results are compared with other conventional DR approaches. After considerable reduction of the raw measurements, a phasor metric for identification of coherency groups of generators is proposed. The recommended measure stands for its simple implementation, interpretation and fast computation. To illustrate the effectiveness of the clustering approach and the coherency of the metrics, a particular study case following the outage of a representative generation unit in France is presented.
\end{abstract}

Index Terms-Power system dynamics, data analytics, coherency groups, frequency problems.

\section{INTRODUCTION}

Access to data measure from more sophisticated monitoring devices such as Phasor Measurement Units (PMUs) allow the transmission system operators (TSO) to have better observability of the extra high voltage networks. With these information it is possible to monitor, in real time or with offline recorded events that jeopardize the safety operation of the power system. In a typical transmission system, where thousands of buses are monitored for control and operation, large volumes of data are produced and stored in local servers requiring significant amounts of central processing unit (CPU) storage. This Big Data problem, present in various other industrial environments, has captured particular attention within the power system community. Synchronized measurements are characterized by large sets of complex, vast and variable data where special considerations are required in order to extract patterns and valuable information among the universe of measurements [1]. To overcome this issue, linear and nonlinear dimensionality reduction (DR) techniques have been used in the literature. Some of the most popular DR methods include principal component analysis (PCA), isometric feature mapping (Isomap) and locally linear embedding (LLE). These approaches have been successfully applied for finding useful low dimensional presentations of high dimensional data; they have been particularly used to solve image processing issues [2], acoustic problems [3] and identification of genome gene patterns [4], among others. The drawback of the aforementioned methods is that it becomes challenging to visualize the final results (dendrograms) when the volume of data is too large, particularly when there are wide variety of information within the data and are not useful for nonlinear datasets.

In this work is presented a nonlinear DR algorithm referred as t-Distributed Stochastic Neighbour Embedding (t-SNE) [5] [6], which is an extension of the plain Stochastic Neighbour Embedding (SNE) approach previously introduced on [7] [4]. Although, these promising nonlinear approaches have been already applied in different domains, these algorithms have not been fully explored in the context of transmission systems of electric power networks. The information provided by wide area monitoring (WAMs) devices such as recorded data from phasor measurement units (PMUs) is considered a special Big Data problem. WAMs include Big Data characteristics such as: volume: require considerable amounts of storage, variety: twodimensional structure and variable length, velocity: fast collection (10-40 ms) and value: accurate and continuous data. To show the effectiveness of the proposed DR algorithm, a novel data analytic method for identification of coherency groups of generators is presented. Frequency measurements of synchronous machines in different regions of the electric power system in continental Europe are used as input. The main contribution of the proposed approach, from a dynamic point of view, is that as consequence of the clustering process, the observability space (number of monitored locations) is drastically reduced to a few machines of a given cluster in order to monitor the dynamic behaviour of the complete system.

The paper is organized as follows; section II introduces the data analytic challenge. Section II.A presents in detail the mathematical description of the proposed DR approach. Section II.B gives a procedure to measure the reliability of the DR method. Section III provides an insight of the dynamic model used to validate the effectiveness of the proposed approach. Section IV presents and compares the simulation results. Section V introduces the novel phasor metric for identification 
of coherency groups and finally, section VI summarize and concludes the results of this work.

\section{FORMULATION OF THE PROBLEM}

. Consider that a spatio-temporal frequency data defined as $\boldsymbol{X} \in \mathbb{R}^{n \times m}$, is constituted by $n$ vectors $\boldsymbol{x}_{i}$ that come from different node frequency measurements. At this point, a set of observation data is created, as depicted in equation (1):

$$
X=\left\{\begin{array}{lllllll}
x_{1} & x_{2} & x_{3} & \ldots & x_{i} & \ldots & x_{n}
\end{array}\right\}^{T}
$$

Considering that the input data generally contains redundant or correlated information, this characteristic is used to obtain a low-dimensional model $\boldsymbol{Y}$, that allows visualize the $K$ clusters immerse into the input data $\boldsymbol{X}$. In this paper, a DR algorithm known as t-Distributed Stochastic Neighbour Embedding ( $t$ $\mathrm{SNE}$ ) [8] is introduced to obtain a reduced model, defined as in equation (2):

$$
\boldsymbol{Y}=\left\{\begin{array}{llll}
\boldsymbol{y}_{1} & \boldsymbol{y}_{2} & \ldots & \boldsymbol{y}_{d}
\end{array}\right\}^{T}
$$

Where the $\boldsymbol{Y} \in \mathbb{R}^{n \times d}$ matrix represents the most relevant information extracted from the input matrix $\boldsymbol{X}$, where $d<n$.

\section{A. $t$-Distributed Stochastic Neighbour Embedding ( $t$-SNE)}

$\mathrm{t}$-SNE is one of the most modern introduced technique to obtain a low reduced data model. t-SNE measures the probability of the data set being neighbours, allowing to improve significantly the cluster visualization as was shown in diverse applications [4], [5], [6], [7], [9].

Mathematically, t-SNE operates by converting the frequency measurements data into a probability distribution over pairs of profiles so similar pairs have a high probability of being picked. The t-SNE starts by converting Euclidean distances into conditional probabilities $p_{i / j}$, as shown in equation (3) below:

$$
p_{i / j}=\frac{\exp \left(-\left\|x_{i}-x_{j}\right\| / 2 \sigma^{2}\right)}{\sum_{k \neq l} \exp \left(-\left\|x_{i}-x_{j}\right\| / 2 \sigma^{2}\right)}
$$

where $p_{i / j}$ marks the probability that $\boldsymbol{x}_{i}$ chooses $\boldsymbol{x}_{j}$ as its neighbor through a Gaussian function centered in the $\boldsymbol{x}_{i}$ point and $\sigma$ is a tuning parameter that controls the bandwidth of the Gaussian kernel around point $\boldsymbol{x}_{i}$. To improve the minimization process, t-SNE redefines this conditional probability [8] as follows:

$$
p_{i j}=\frac{p_{i / j}+p_{j / i}}{2 n}
$$

This redefinition, allows symmetrized the measure of similarity between the data sets $\boldsymbol{x}_{i}$ and $\boldsymbol{x}_{j}$ in the high dimensional space. Notice that the perplexity $\sigma$ is a free parameter that can be thought of as the number of effective neighbours and it can be set by experience on the data for clustering or following the search procedure defined in [8], [10].

Subsequently, the SNE calculate the output space coordinates with a conditional probability $q_{i / j}$; therefore, tSNE computes the probabilities inspired by the Student $t$ distribution with one degree of freedom to model the similarity between data $\boldsymbol{y}_{i}$ and $\boldsymbol{y}_{j}$ in a low-dimensional space and relieving the "Crowding Problem" with this change. Likewise, the similarity between the corresponding low-dimensional models of these frequency measurements sets is defined in equation (5) as:

$$
q_{i j}=\frac{\left(1+\left\|\boldsymbol{y}_{i}-\boldsymbol{y}_{j}\right\|^{2}\right)^{-1}}{\sum_{k \neq l}\left(1+\left\|\boldsymbol{y}_{i}-\boldsymbol{y}_{j}\right\|^{2}\right)^{-1}}
$$

Based on the fact that the map points $\boldsymbol{y}_{i}$ and $\boldsymbol{y}_{j}$ correctly model the similarity between the high-dimensional data points $\boldsymbol{x}_{i}$ and $\boldsymbol{x}_{j}$, we can deduce that the probabilities $p_{i j}$ and $q_{i j}$ will be equal. Further, assuming a faultless model, the lowdimensional map $\boldsymbol{Y}$ of frequency measurements can obtained from a minimization procedure of Kullback-Leibler function, which is defined as follow in equation (6):

$$
C=K L\left(P_{i} \| Q_{i}\right)=\sum_{i} \sum_{j \neq i} p_{i j} \log \frac{p_{i j}}{q_{i j}}
$$

where $C$ is the cost function that measure the divergence between two probability distributions. The gradient descent method is used to minimize the difference between the corresponding $p_{i j}$-values and $q_{i j}$-values involved on the cost function depicted on equation (7). Mathematically, the gradient update with a momentum term is given by

$$
\gamma^{(t)}=\gamma^{(t-1)}+\eta \frac{\partial C}{\partial \gamma}+\alpha(t)\left(\gamma^{(t-1)}-\gamma^{(t-2)}\right),
$$

where $\gamma^{(t)}$ indicate the solution at iteration $t, \eta$ is the learning rate that weight the gradient cost function

$$
\frac{\partial C}{\partial y_{i}}=4 \sum_{j \neq i}^{p}\left(p_{i j}-q_{i j}\right)\left(\boldsymbol{y}_{i}-\boldsymbol{y}_{j}\right)\left(1+\left\|\boldsymbol{y}_{i}-\boldsymbol{y}_{j}\right\|^{2}\right)^{-1}
$$

To improve the convergence and the optimization solution process, the momentum term $\alpha(t)$ is used to avoid poor local minima [8].

\section{B. Cluster Validation}

The number of clusters and its labels are not provided by the DR technique, so the determination of the optimal number of clusters results essential for the clustering analysis. To carry out this procedure, the Silhouette cluster validation index was 
selected based on its ability for examining compactness and separation of the clusters [11]. We now present the formal definitions of this cluster's validation index. Let $\boldsymbol{Y}=$ $\left\{\begin{array}{llll}\boldsymbol{y}_{1} & \boldsymbol{y}_{2} & \ldots & \boldsymbol{y}_{d}\end{array}\right\}^{T}$ be the data set and let $\boldsymbol{C}=\left\{\begin{array}{llll}c_{1} & c_{2} & \ldots & c_{K}\end{array}\right\}^{T}$ be its clustering into $K$ clusters, and the distance between vectors of reduced model data $\boldsymbol{y}_{i}$ and $\boldsymbol{y}_{k}$ be defined as $d\left(\boldsymbol{y}_{i}, \boldsymbol{y}_{k}\right)$. The Silhouette index $(S I)$ assigns a quality measure to each data point in $c_{K}$ known as the silhouette width. The silhouette width is a confidence indicator on the membership of the $i$-th data vector in cluster $c_{k}$ and is defined as follow:

$$
S I(i)=\frac{b(i)-a(i)}{\max \{b(i), a(i)\}}
$$

where $a(i)=\frac{1}{m_{j}-1} \sum_{\substack{k=1 \\ k \neq i}}^{m_{j}} d\left(\boldsymbol{y}_{i}, \boldsymbol{y}_{k}\right)$ is the average distance between the $i$-th vector $c_{j}$ and the other vectors in the same cluster $c_{k}$ and $b(i)=\min _{\substack{n=1, \ldots, K \\ n \neq j}}\left\{\frac{1}{m_{n}} \sum_{k=1}^{m_{n}} d\left(\boldsymbol{y}_{i}, \boldsymbol{y}_{k}\right)\right\}$ is the minimum average distance between the $i$-th vector in the cluster $c_{j}$ and all vector clustered in the cluster $c_{k}$. From expression (8), it may be inferred that the $S I(i)$ value will take values between $-1 \leq S I(i) \leq 1$.

The clustering algorithm operates a number of times for different number of clusters each time. In this research, this action is carry out for the $K$-means algorithms. Then, we outline the respective curve of the validation index and search for the significant "knee" within the diagram. The number of clusters at which the "knee" is observed indicates the optimum clustering for the selected data set. The index also is entitled to obtain its corresponding labels, which permit evaluate the quality of the separation provided initially by the DR technique.

\section{General Procedure}

The proposed procedure allows to evaluate the performance of the presented t-SNE technique as well as provide a framework for the comparison with other conventional DR techniques in terms of clusters visualization, clustering data and potential application for power system stability assessment. Figure 1 shows a representation of the architecture of LIS Analytics \& Visualization. This platform has been developed to optimize reliability and fast query retrieval.

\section{STUDY CASE}

The model under investigation is the initial dynamic model of ENTSO-E. This network is a comprehensive representation of the interconnected Continental European power system and is depicted on Figure 2; it includes 26 areas or countries highlighted in green, from which France, Germany, Italy, Greece, Turkey and Spain are the most detailed regions as indicated by the larger number of elements.

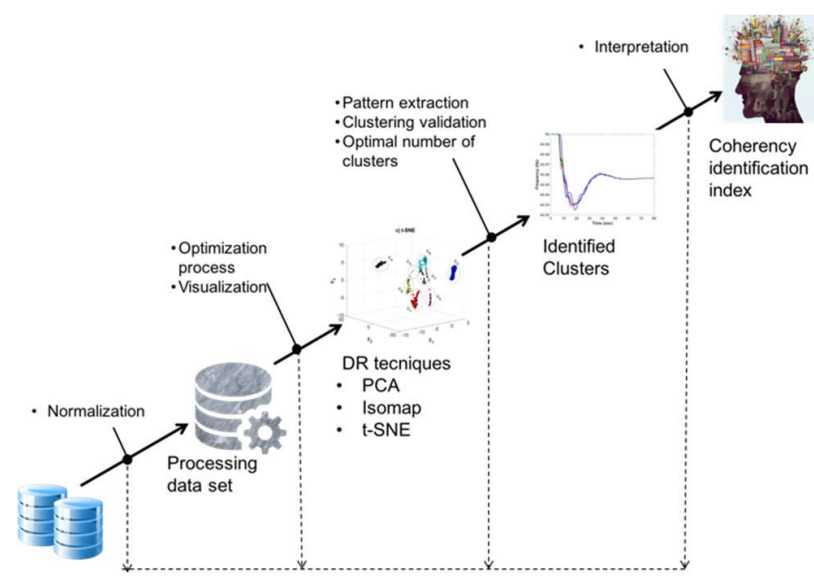

Figure 1 Outline of the proposed general procedure.

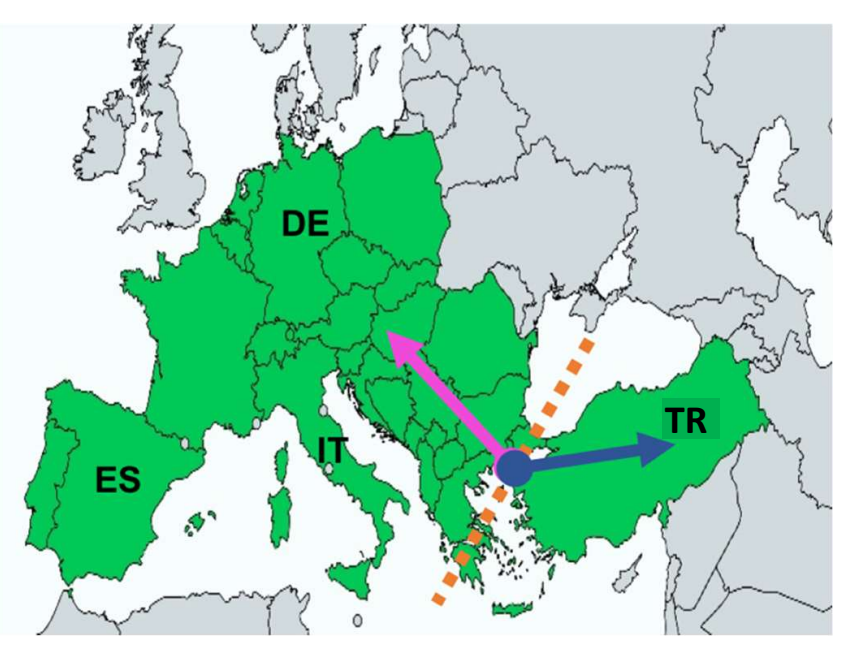

Figure 2 The interconnected network of ENTSO-E (green), highlighting the countries where measurements were taken (Section IV) and illustrating with arrows the most relevant coherency groups (Section V).

More details about this mathematical model can be found on [12] and [13]. The complexity and precision of the system is evident as indicated by the large volume of components involved. There are more than 18000 transmission lines connecting devices spanned along extended distances from up to $300 \mathrm{~km}$ in Turkey to short connections of $0.1 \mathrm{~km}$ in the Netherlands. Regarding connecting buses or nodes, there is a distribution among various voltage levels including less than $20 \mathrm{kV}$ up to $400 \mathrm{kV}$. Concerning the system loads, more than $50 \%$ of them are smaller than $40 \mathrm{MW}$ and with respect to generators installed in the system, from the more than 10,000 generators available, only 1,078 have a rated power larger than 250 MVA and are represented by a sixth order round rotor synchronous machine model. Each of them equipped with a standard governor (TGOV1) model, a standard automatic voltage regulator (SEXS) and a standard power system stabilizer (PSS2A). 


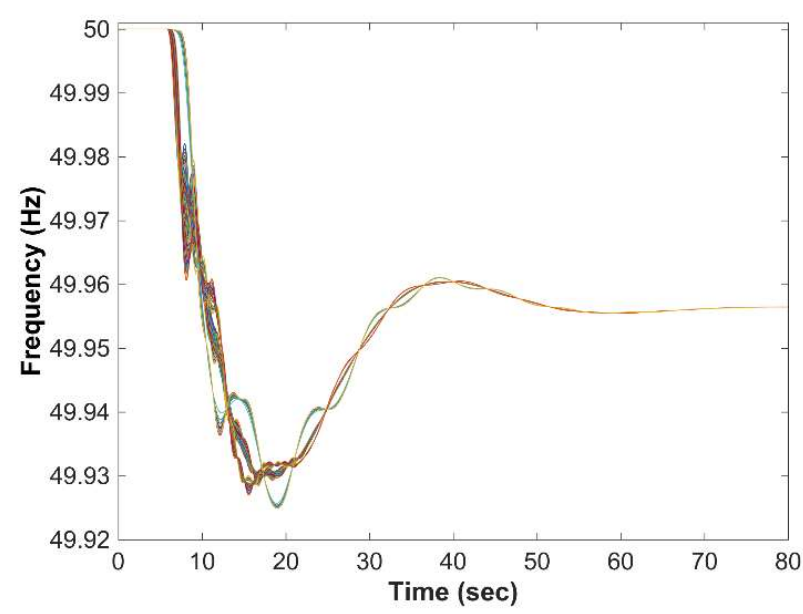

Figure 3 Frequency of synchronous machines in DE, IT, ES and TR following the outage of one generation unit in France.

\section{APPLICATION TO THE ENTSO-E MODEL}

To validate the effectiveness of the proposed approach, the ENTSO-E initial dynamic model of continental Europe, described in the previous section, was used for investigation. Figure 3 depicts the set of data used as input in dimensionality reduction (DR) techniques such as the one proposed on Section II.A.

The measurements on Figure 3 represent the frequency of more than 600 synchronous machines, following the outage of a representative generation unit in France, in 4 different countries: Germany (DE), Italy (IT), Turkey (TR) and Spain (ES). These countries where selected for having the larger number of synchronous generators used to model each region and to have a wide observation of the frequency variations in entire system. Table 1 displays the precise number of elements existing on each area and the total amount of active power (in GW) per area.

Following the procedure presented on Section II.B, the first step, in order to gain insight on the grouping analysis, is to calculate the normalized average clusters, which means to calculate the optimal number of groups to be used by the selected DR approach. In this case, we have compared the results of applying the t-SNE method against the popular and widely used principal component analysis (PCA) and Isomap, more details about these methods can be found in [9].

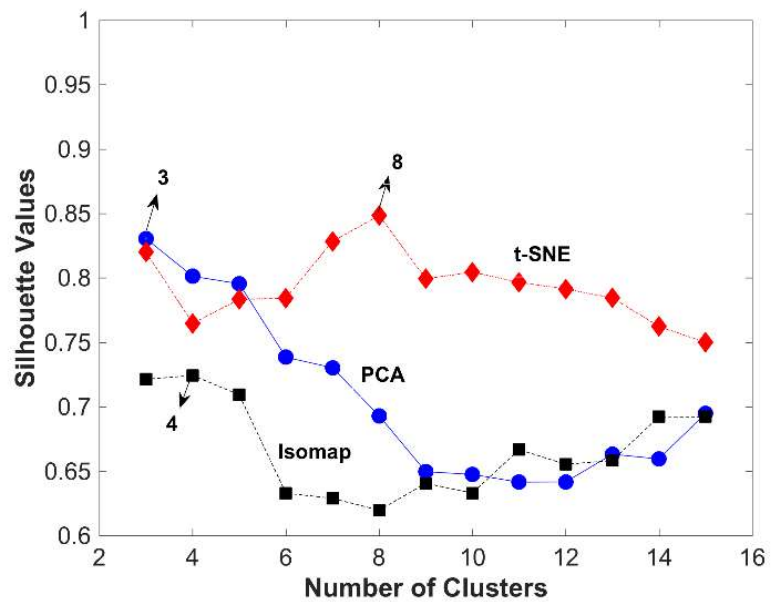

Figure 4 Determination of optimal number of clusters using the Silhouette index.

Table 1 Number of synchronous generators per country and their total active power generation in GW

\begin{tabular}{|c|c|c|c|c|}
\hline & $\begin{array}{c}\text { Germany } \\
\text { (DE) }\end{array}$ & $\begin{array}{c}\text { Italy } \\
\text { (IT) }\end{array}$ & $\begin{array}{c}\text { Spain } \\
\text { (ES) }\end{array}$ & $\begin{array}{c}\text { Turkey } \\
\text { (TR) }\end{array}$ \\
\hline Num. Gen & 292 & 144 & 70 & 127 \\
\hline Total GW & 53.014 & 20.241 & 26.195 & 36.403 \\
\hline
\end{tabular}

The results of calculating the optimal number of clusters are depicted on Figure 4, where the "knee" is associated to the largest number of each trace indicating the optimal number of clusters for a given DR method. The normalized average cluster is defined as follows:

$$
c_{i}=\frac{1}{h} \sum_{j=1}^{h} x_{j}(t, v)
$$
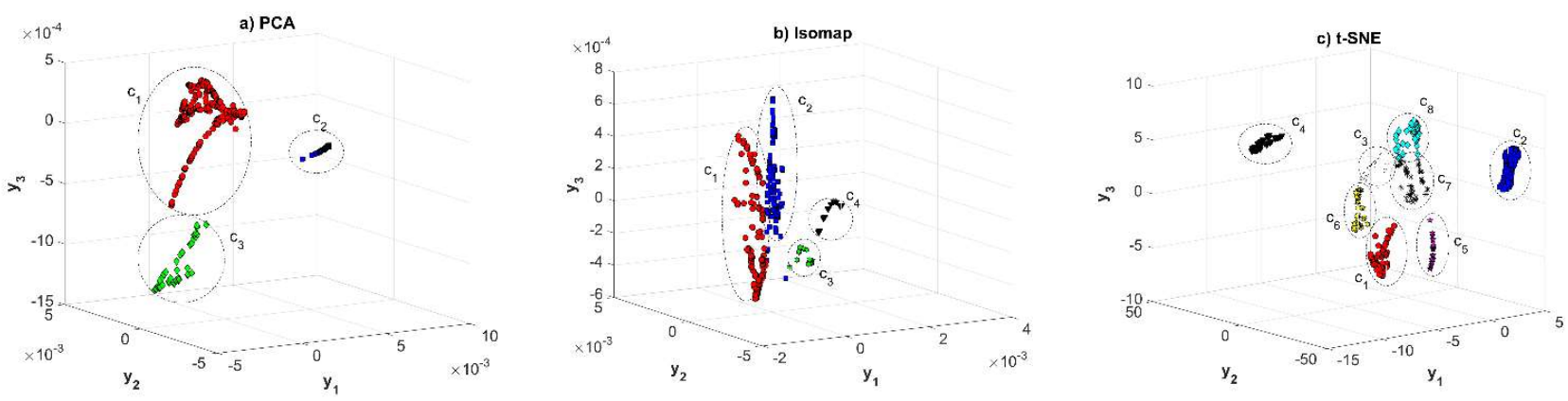

Figure 5 Scatter 3-D plot of the optimal number of clusters. 

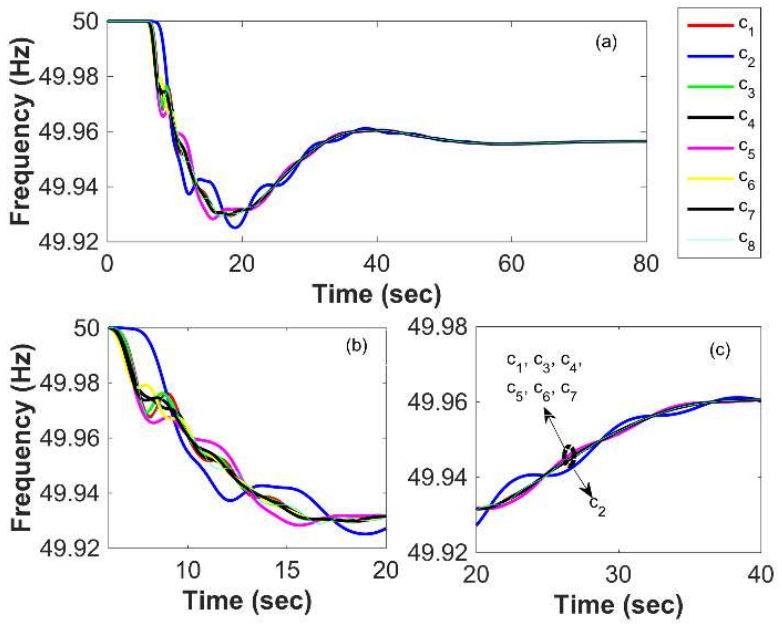

Figure 6 Average clusters identified by t- SNE

where $h$ denotes the number of signals that composed each cluster. The corresponding labels $v$ are provided by the validation index defined in the equation (10). From Figure 4, it can be seen that the optimal number of groups are 3 for PCA, 4 for Isomap and 8 for t-SNE, respectively. After selecting the optimal number of clusters, 3 different DR approaches were applied to the same set of data and the results are depicted as scatter 3D plots on Figure 5-(a), (b) and (c), respectively. From the results of the $3 \mathrm{D}$ plots, it can be observed that, for the same set of data, other classical DR methods such as PCA and Isomap detect fewer number of groups compared to t-SNE. The results suggest that $\mathrm{t}-\mathrm{SNE}$ provide more accuracy identifying smaller subgroups that exist when the variation of the input data is much smaller, while the other DR methods cannot. To further analyse the results of applying t-SNE, Figure 6 display the reduced set of data highlighting the colour code of the clusters and Figure 7 its corresponding geographical location, as indicated on Figure 5-(c). In addition, Table 2 displays the specific number of machines participating on each cluster and their geographical location. From the results depicted on Figure 6-(a) and Figure 7-(a), it can be distinguished three dynamic behaviours: cluster $c_{2}$, represented in blue and constituted mainly by Turkey, against cluster $c_{5}$ and the rest of the machines. Furthermore, to illustrate that the t-SNE approach presents higher accuracy compared to the other methods, Figure 6-(b) and (c) depict a close up of the clusters evolution over two windows of 20 seconds each. Figure 6-(b) and 7-(b) display the first $20 \mathrm{sec}$ block $(0-20 \mathrm{sec})$. In these figures, it can be observed the results of the first fourteen seconds after the fault has been cleared (6$20 \mathrm{sec}$ ), there are eight clusters with opposite phase oscillating against each other. Moreover, Figure 6-(c) and 7-(c) present the close up of the second block $(20-40 \mathrm{sec})$. In these figures, it is show that after the controls respond to attenuate the frequency unbalance, a different dynamic behaviour of the clusters can be observed. Figure 6-(c) and 7-(c) show mainly two groups: $c_{2}$ against the rest of the clusters.

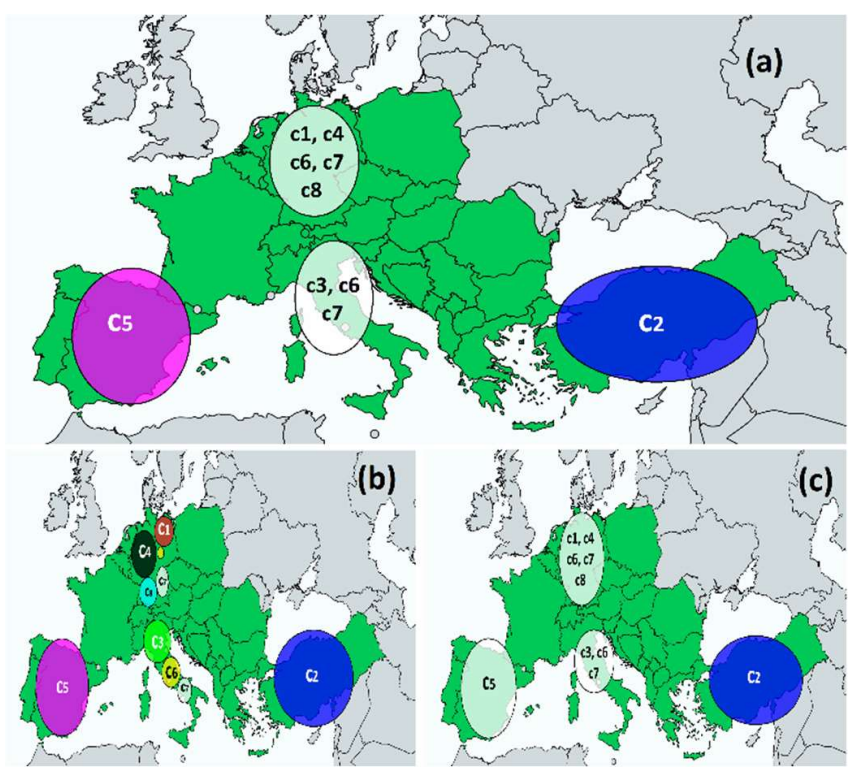

Figure 7 Geographical location of average clusters.

Table 2 Number and geographical location of the synchronous generators involve on each cluster

\begin{tabular}{|c|c|c|c|c|}
\hline \# Clusters & Germany & Italy & Spain & Turkey \\
\hline $\boldsymbol{c}_{\boldsymbol{1}}$ & 63 & $\mathrm{x}$ & $\mathrm{X}$ & $\mathrm{X}$ \\
\hline $\boldsymbol{c}_{\boldsymbol{2}}$ & $\mathrm{x}$ & $\mathrm{x}$ & $\mathrm{X}$ & 127 \\
\hline $\boldsymbol{c}_{\boldsymbol{3}}$ & $\mathrm{x}$ & 62 & $\mathrm{X}$ & $\mathrm{X}$ \\
\hline $\boldsymbol{c}_{\boldsymbol{4}}$ & 90 & $\mathrm{X}$ & $\mathrm{X}$ & $\mathrm{X}$ \\
\hline $\boldsymbol{c}_{\mathbf{5}}$ & $\mathrm{x}$ & $\mathrm{X}$ & 70 & $\mathrm{X}$ \\
\hline $\boldsymbol{c}_{\boldsymbol{6}}$ & 17 & 49 & $\mathrm{X}$ & $\mathrm{X}$ \\
\hline $\boldsymbol{c}_{\boldsymbol{7}}$ & 51 & 33 & $\mathrm{X}$ & $\mathrm{X}$ \\
\hline $\boldsymbol{c}_{\boldsymbol{8}}$ & 71 & $\mathrm{x}$ & $\mathrm{X}$ & $\mathrm{X}$ \\
\hline
\end{tabular}

V. IDENTIFICATION OF COHERENCY GROUPS

In this section, two metrics used to identify the participation of the generators in relationship of the clusters that have been found are presented. These metrics where develop to provide simple implementation, easy interpretation and fast computation. They give a quantitative measure of the interaction among the generators and are expressed in the form of magnitude and phase angle as described next.

\section{A. Participation Measure}

The proposed measure in this section is based on the integral of the difference among the clusters and a reference, which in this case is the median. Equation (11) presents the calculation of the median $(M)$ :

$$
M(t)=L+\left(\frac{\frac{N}{2}-F(t)}{f_{m}}\right) C
$$

where $L$ is the lower boundary of the median, $N$ is the number of data, $F$ is the frequency before the median, $f_{m}$ is the total frequency on the median and $C$ is the average of the data. After 
$M(t)$ has been calculated, the participation measure $\left(P M_{i}\right)$ for the corresponding cluster $i$ is calculated as follows

$$
\begin{gathered}
\tau_{i}(t)=M(t)-c_{i}(t) \\
P M_{i}=\frac{1}{\sup \rho_{i}} \int_{t_{1}}^{t_{2}} \tau_{i}(t) d t, \quad i=1, \cdots, n .
\end{gathered}
$$

where $c_{i}(t)$ is the time series of the $i$ cluster and $\sup \rho_{i}$ is the maximum absolute value of the interval $\left(t_{1}, t_{2}\right)$. Figure 8 -(a) and (b) depict the bar plots of the participation measures for both of the intervals displayed on Figure 6-(b) and (c), respectively. The results depicted on Figure 8 reinforce the outcomes of the analysis in Section IV. The length of the bars on Figure 8 provide a direct quantitative measure of how much each cluster associate with a given event in the system as well as provide stability system information. From Figure 8-(a), three different coherency groups of generators can be identified as follows: group $G_{1}$, comprised by the cluster $c_{2}$ (blue bar) which includes all synchronous machines in Turkey, group $G_{2}$ represented by cluster $c_{5}$ or Spain and the third group $G_{3}$, which comprehend the rest of the machines with combinations of subgroups in Germany and Italy (see Table 2).
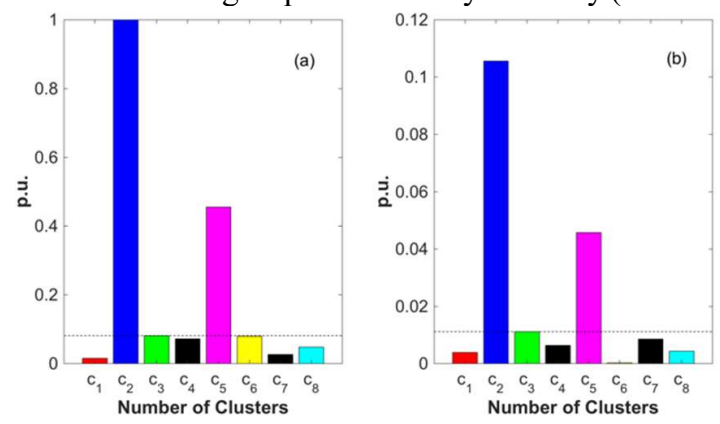

Figure 8 Participation measures and definition of coherency groups for (a) the clusters during the first 20 seconds and (b) the clusters during the 20-40 sec period.
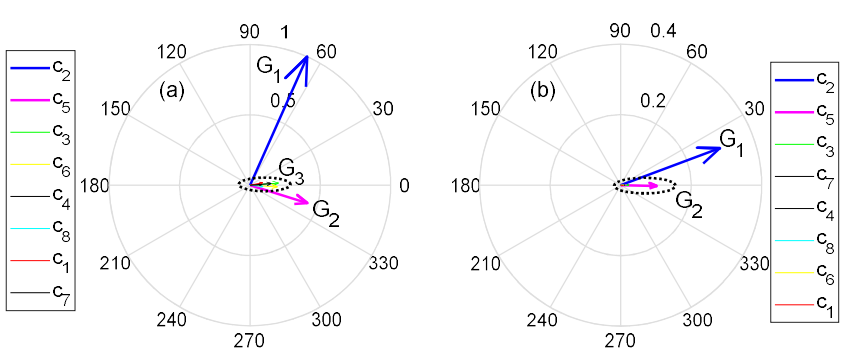

Figure 9 Phase angle and coherency groups of clusters (a) during the first 20 seconds and (b) the clusters during the $20-40 \mathrm{sec}$ period.

Similarly, Figure 8-(b) display the participation measures for the interval from 20-40 sec. In this subplot, a different dynamic behaviour of the clusters can be observed. The coherency groups $G_{2}$ and $G_{3}$ from the first $20 \mathrm{sec}$, are merged together creating a larger coherency subgroup. In other words, after 20 sec, the generators in Turkey oscillate against the generators in Italy, Germany and Spain (Figure 2). This can be concluded from the marginal participation of most the clusters, as depicted on Figure 8-(b).

\section{B. Phase of Signals}

To complement the participation measures $(P M)$ introduced before, a phase angle that provides information about the relationship among the clusters, is calculated as follows:

$$
\begin{gathered}
\tilde{\theta}_{i}(t)=\left\{\left[\tan \left(\tau_{i}(t)\right)\right] / \sup \beta_{i}\right\} \times \pi \\
\theta_{i}=\frac{1}{n} \sum_{t=1}^{n} \tilde{\theta}_{i}(t)
\end{gathered}
$$

where $\widetilde{\theta}_{i}$ is the tangent in radians of the difference between the median and each cluster, $\sup \beta_{i}$ is the maximum absolute value of the tangent. Note that tangent $\tilde{\theta}_{i}(t)$ in equation (13) is calculated for each sampling time over the entire interval, if $\tilde{\theta}_{i}(t)$ is equal to zero the signal is in phase with the reference for a particular instant. $\theta_{i}$ is the average angle of the interval and gives a global measure of the phase shift for a given section. To illustrate this concept, the results of applying equation (13) to the signals shown on Figure 6-(b) and (c), are depicted in compass plots displayed on Figure 9-(a) and (b). Note that the vectors plotted here, were formed using the magnitudes from the participation measures and the global phase angles as follows:

$$
\vec{c}_{i}=P M_{i} \angle \theta_{i}
$$

Figure 9-(a) present the phase angle and the coherency groups during the first 20 seconds. Correspondingly, Figure 9-(b) displays the vectors and the reduced coherency groups during the second period $(20-40 \mathrm{sec})$. From the results, it can be noticed that initially, the phase angle difference was significantly larger among the groups, clearly defining the existing coherent groups. Furthermore, after the controllers responded, beyond $20 \mathrm{sec}$, the phase angle reduces considerably and the number of coherent groups become smaller. The results shown on Figures 6, 7 and 8 suggest the onset of low-frequency oscillations. To verify this observation a spectral signal decomposition analysis was carried out. For this reason, the Fast Four Transform (FFT) was selected to process the original data set related with frequency measurements $(\boldsymbol{X})$ and the data set obtained from the clustering process $\left(c_{i}, i=1, \ldots, n\right)$.

Figure 10 shows the spectral decomposition for both windows under investigation. The FFT applied to the first window of study $(0-20 \mathrm{sec})$ is displayed on Figure 10-(a) to (e). From these suplots it can be observed the presence of several low frequencies oscillatory modes: $0.09,0.2,0.30$ and $0.4 \mathrm{~Hz}$. Furthermore, after applying FFT to the second window under investigation (20-49 sec), only two low frequency oscillatory modes $(0.09$ and $0.2 \mathrm{~Hz})$ are observed as depicted on Figure 10-(f) to (j). 

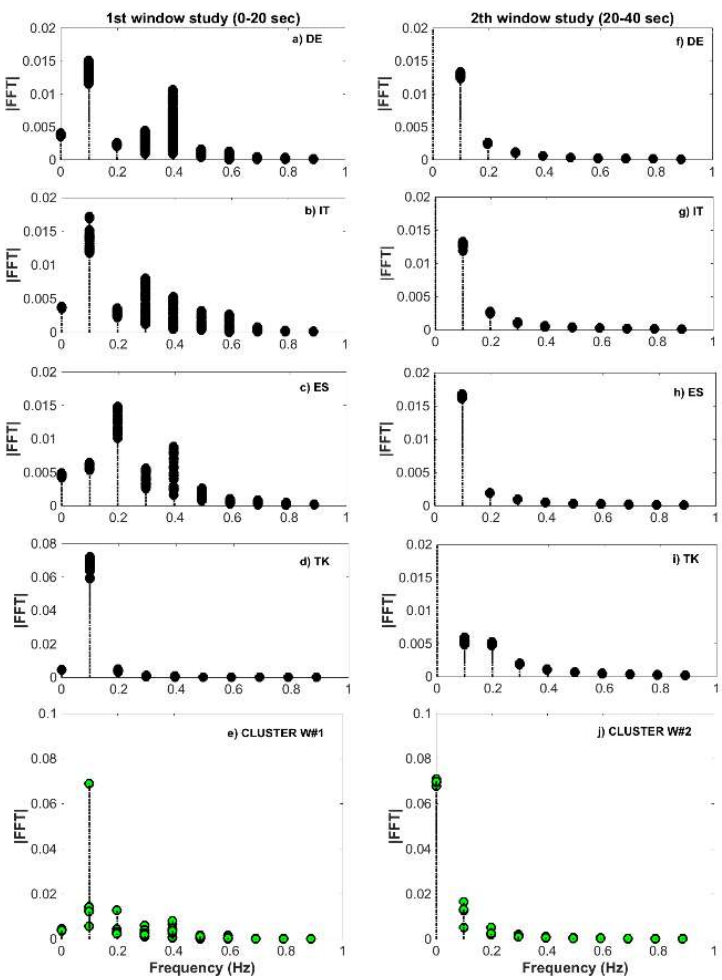

Figure 10 Spectral decomposition of data sets $X$ and $C_{i}$ for two window of study: (a-e) during the first 20 seconds and (f-j) the clusters during the 20-40 sec period.

The low frequency modes identified in this study are in agreement with the low frequency modes characterized on diverse previous studies on the ENTSO-E system [14], [15]. Finally, it should be noteworthy that, the identified clusters by the proposed t-SNE approach preserve the original oscillatory mode content, which confirms the potential of this proposal on transmission power system problems.

\section{CONCLUSION}

In this paper a DR technique, referred as the t-Stochastic Network Embedding (t-SNE), for clustering of Big Data in power systems has been presented. The proposed DR approach has been compared with other conventional techniques such as PCA and Isomap. Frequency measurements of the ENTSO-E dynamic model have been used as inputs in the DR methods. From the results, it has been observed that t-SNE can identify clusters even when the variation of data is small, while PCA and Isomap cannot. These findings suggest that in the future an online clustering technique may be useful to evaluate the clustering evolution. Also, a novel approach for identification of coherency groups has been proposed. The metric provides a global participation factor and a phase angle of the clusters in relation to a given contingency. The new metric stands for its simple implementation and fast computation.

\section{AKNOLEGEMENTS}

The authors acknowledge the Swiss National Science Foundation (SNSF) under the Ambizione Energy Grant and the Swiss Federal Office of Energy (BFE). This research is also part of the activities of SCCER FURIES, which is financially supported by the Swiss Innovation Agency (InnosuisseSCCER program). Finally, the project has also received funding in the framework of the joint programming initiative ERA-Net Smart Grids Plus, with support from the European Union Horizon 2020 research and innovation program.

\section{REFERENCES}

[1] S. E. Haupt und B. Kosović, «Variable Generation Power Forecasting as a Big Data Problem,» IEEE Transactions on Sustainable Energy, Bd. 8, Nr. 2, pp. 1949-3029, April 2017.

[2] R. Wang, F. Nie, R. Hong, X. Chang, X. Yang und W. Yu, «Fast and Orthogonal Locality Preserving Projections for Dimensionality Reduction,» IEEE Transactions on Image Processing, Bd. 26, Nr. 10, pp. 5019-5030, 2017.

[3] A. Bartkowiak und R. Zimroz, «Dimensionality reduction via variables selection - Linear and nonlinear approaches with application to vibration-based condition monitoring of planetary gearbox,» Applied Acoustics, Bd. 77, Nr. 3, pp. 169-177, 2014.

[4] A. Mahfouz, G. v. d. Martijn, M. v. d. Laurens, S. Huisman, M. Reinders, M. J. Hawrylycz und B. P.F., «Visualizing the spatial gene expression organization in the brain through non-linear similarity embeddings,» Methods, Bd. 73, Nr. 1046-2023, pp. 79 - 89, 2015.

[5] K. k. Tam, Visualizing breast cancer data with t-SNE, Nijmegen, the Netherlands: Radboud University, October 25, 2013.

[6] J. Cheng, H. Liu, F. Wang, H. Li und C. Zhu, «Silhouette Analysis for Human Action Recognition Based on Supervised Temporal t-SNE and Incremental Learning,» IEEE Transactions on Image Processing, Bd. 24, Nr. 10, pp. 3203-3217, 2015.

[7] D. Lunga und O. Ersoy, «Spherical Stochastic Neighbor Embedding of Hyperspectral Data,» IEEE Transactions on Geoscience and Remote Sensing, Bd. 51, Nr. 2, pp. 857-871, 2013.

[8] L. v. d. Maaten und G. Hinton, «Visualizing Data using t-SNE,» Journal of Machine Learning Research, pp. 2579-2605, 2008.

[9] A. Arechiga, E. Barocio, J. Ayon und H. Garcia, «Comparison of Dimensionality Reduction Techniques for Clustering and Visualization of Load Profiles,» IEEE PES T\&D-LA, in Proc., 2016.

[10] Y. Cao und L. Wang, "Automatic Selection of t-SNE Perplexity,» Automatic Machine Learning Workshop ICML , pp. 1-7, 10 August Sidney, 2017.

[11] K. L. und P. J. Rousseeuw, Finding Groups in Data: An Introduction to Cluster Analysis. Hoboken, John Wiley \& Sons, 1990.

[12] A. Semerow, S. Hhn, M. Luther, W. Sattinger, H. Abildgaard, A. D.Garcia und G. Giannuzzi, «Dynamic study model for the interconnected power system of continental Europe in different simulation tools,» PowerTech, 2015 IEEE Eindhoven,, In Proc., 2015.

[13] F. R. Segundo, P. Korba, K. Uhlen, E. Hillberg, G. Lindahl und W. Sattinger, «Evaluation of the ENTSO-E Initial Dynamic Model of Continental Europe Subject to Parameter Variations,» IEEE ISGT North America, pp. 315-320, in Proc., 2017.

[14] E. Grebe, J. Kabouris, S. L. Barba, W. Sattinger und W. Winter, «Low frequency oscillation in the interconnected system of continental Europe,» IEEE-PEE General Meeting, 25-29 July RI USA, 2010.

[15] O. Tor, C. Gencoglu, O. Yilmaz, E. Cebeci und A. N. Guven, «Damping measures against prospective oscillations between Turkish grid and ENTSO-E System,» IEEE, Power system Technology (POWERCON), 24-28 Octuber Hangzhou, China, 2010. 